$\xi$

\title{
Linear array Yagi-Uda 5G antenna for vehicular application
}

\author{
K. Sreelakshmi ${ }^{1 *}$, Pronami Bora ${ }^{2}$, Mona Mudaliar ${ }^{3}$, Yuvraj Baburao Dhanade ${ }^{4}$, B.T.P Madhav ${ }^{5}$ \\ ALRC, Department of ECE, Koneru Lakshmaiah Education Foundation, Ap, India \\ *Corresponding author E-mail: sreelakshmi@kluniversity.in
}

\begin{abstract}
A two element $2 \mathrm{X} 2$ linear array Yagi antenna which is meant to operate at frequency of $3.5 \mathrm{GHz}$ which can be used for low band $5 \mathrm{G}$ as well as for WIMAX is presented in this paper. This array consists of two radiating elements of eight parasitic elements each with overall dimensions of $110 \times 60 \times 1.6 \mathrm{~mm} 3$. The proposed antenna was built on Rogers Duroid substrate. It exhibits a good bandwidth (impedance bandwidth of single antenna element about 600Mhz.for S11 less than-10dB at the center frequency of $3.5 \mathrm{GHz}$ ) and gain of $10.5 \mathrm{~dB}$. The idea behind this paper is to attain good gain along with bandwidth which can be utilized for vehicular applications.
\end{abstract}

\section{Introduction}

One of the most convenient and luxury style of transport is by car, so it has become necessary to implement all types of luxurious entertainment, driver information and communication systems in a vehicle. The next generation of wireless communication which is Fifth generation or $5 \mathrm{G}$ holds a high rate of adoption in automobile industry as it provides better communication and different unique applications like multi person video calling, autonomous driving, real time gaming and many more on go, which present $3 \mathrm{G}$ or $4 \mathrm{G}$ cannot provide. Because of its low latency and high speed it can be enabled in vehicles to communicate with outside world and nearby vehicles which could result in significantly more efficient and well organized traffic system. If all of the vehicles on a road and road side objects were connected to form a network, they can share safety information as a part of incorporating a Intelligent traffic management system. Hence Vehicle to vehicle communications can pave way for potentially travel at much higher speeds and within greater proximity of each other without risk of accident. Due to favourable properties the frequency bands for $5 \mathrm{G}$ applications ranges for lower $5 \mathrm{G}$ bands below $6 \mathrm{GHz}$ is $3.4-3.8$ $\mathrm{GHz}$ and 4.4 to $4.9 \mathrm{GHz}$ are deployed for vehicular applications.

It has become utmost important to design an antenna with small size, high gain and broad beam-width coverage for this vehicular communications. Its well known that in antennas used for communications suffer from most common problem which is path loss. To prevail over this problem adopting an antenna array is much suitable solution to increase gain as well as physical aperture.

The first fabricated microstrip Yagi antenna was introduced by Huang [4], which comprises one reflector element, one driven element, and two director elements. That microstrip Yagi antenna was able to achieve a gain of around $8 \mathrm{~dB}$. A typical microstrip Yagi antenna consists of a driven patch element and a few parasitically coupled reflector and director patch elements [5]. It is a well-established fact that the directivity of microstrip Yagi antenna greatly depends on the number of directors [6]. Various at tempts have been made to enhance the gain of Yagi antenna by incorporating more than a single director [7-12].

\subsection{Introduction to proposed antenna}

The conventional Yagi antenna is a directional antenna which is well known for its high directivity and gain but the drawback of it was its size and it is not conformal because of its structure. On the other hand the microstrip antenna which is small in size, light weight and compatible with other devices paves way for fabrication of microstrip quasi Yagi antenna where Yagi antenna like structure is designed on a microstrip antenna which possesses the features of high gain and directivity as the conventional Yagi antenna. it also has the advantages of wide bandwidth, low cost and easy to fabricate.

\subsection{Mechanism of Microstrip Yagi-Uda}

In a Microstrip Yagi -Uda the electromagnetic energy is coupled from the driven element dipole through space into the parasitic dipoles and then gets reradiated to form a directional beam. This mechanism is analogous to conventional Yagi antenna, but the slight difference is that the substrate also plays some role in coupling by surface waves. The microstrip patch usually couples very little energy as it radiates largely in broadside and slightly along the direction of the ground plane [13-17]. To attain maximum behavior like a conventional Yagi-Uda antenna the microstrip patches has to place vey near, the separation should be less in between parasitic patches to attain significant amount of coupling. To attain maximum coupling the spacing between the elements should be equal to or less than the thickness of the substrate and this is experimentally found.[18-20].

In this paper we have proposed a microstrip Yagi antenna for 5G applications in a vehicle because of its high directivity and high gain which is suitable for wireless communications. The proposed antenna is a two element linear array which can obtain a high gain and good bandwidth. The simulated results shows the gain and reflection coefficients attained by antenna. 


\section{Design of single element microstrip Yagi antenna}

The Geometry and detailed dimensions of the proposed antenna element is shown in Fig.1. The proposed Quasi-Yagi antenna is printed on the Rogers Duroid 5880 substrate with dielectric constant of 2.2, conductor loss ( $\tan \delta$ ) of 0.0009 and thickness of 1.6 $\mathrm{mm}$.. The antenna is matched with $50 \Omega$ input impedance. The antenna consists of three main components, a dipole driver, the parasitic directors and the reflector element. The ground plane acts as a reflector to achieve a directive radiation pattern. To further improve the directivity, eight parasitic directors are designed and optimized at the operating frequency of $3.5 \mathrm{GHz}$.

As per design equations of the yagi antenna the length of the driver is $0.5 \lambda_{g}$ and the length of the director is $0.45 \lambda_{g}$ where ' $\lambda_{g}$ ' is the effective wavelength of the antenna and can calculate by

$$
\lambda_{g}=\frac{c}{f r \sqrt{\varepsilon_{r}+1}}
$$

Where $\mathrm{C}$ is the velocity of the Electromagnetic wave of free space, $\varepsilon_{r}$ is the relative dielectric constant of the substrate, $f_{r}$ is the resonant frequency of the antenna. We can calculate the width of the radiators by formula

$$
w=\frac{c}{f r \sqrt{2\left(\varepsilon_{r}+1\right)}}
$$

We can calculate the value of each parameters by formula (1) and (2). Which were shown in table 1.

Table 1: Antenna Dimensions in $\mathrm{mm}$

Table 1: Antenna Dimensions in mm
\begin{tabular}{|l|l|l|l|l|l|l|l|}
\hline L & W & L1 & W1 & Wf & W2 & W3 & W4 \\
\hline 55 & 60 & 20.8 & 11.662 & 2.7 & 17.975 & 3.28 & 1 \\
\hline
\end{tabular}

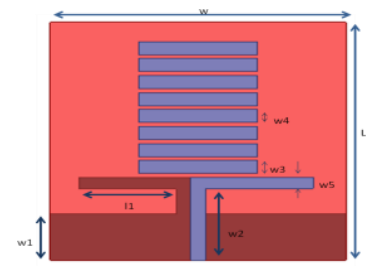

Fig. 1: Proposed Yagi-Uda Antenna Geometry

In this design, the antenna simulations are done using the fullwave electromagnetic field simulator Ansoft HFSS version 13. The Fig.2, depicts the simulated return loss (S11) of the proposed single microstrip Quasi-Yagi antenna. The operating bandwidth is about $620 \mathrm{MHz}$ for S11 less than - 10dB at the center frequency of $3.5 \mathrm{GHz}$ of the antenna can be suitable for array design. The Fig 5, depicts the far field radiation pattern of $\mathrm{E}$ and $\mathrm{H}$ plane of a single antenna element. The antenna peak gain is more than $7 \mathrm{~dB}$. The above single element Yagi antenna yields a Far field gain of more than $7 \mathrm{~dB}$ but in order to acquire more gain from this model the next step of modification is implementing a linear array for the above model. Here we have proposed the 2 element linear array Yagi antenna which can attain better gain, bandwidth and radiation pattern compared to single element.

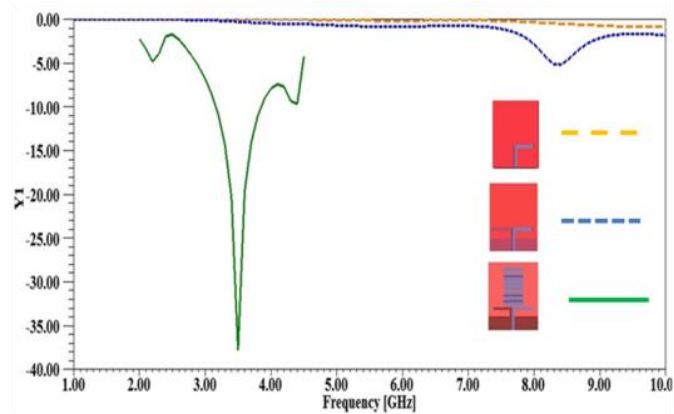

Fig. 2: Step by Step Construction of Yagi Antenna and corresponding S11

\section{Proposed 2 Element Linear Array Yagi- Uda Antenna}

The schematic of the 2 element linear array of the microstrip Quasi-Yagi antenna is shown in Fig.3. In this design, $1 \times 2$ uniform linear array (sub-array) antennas have been used, where each radiating element of them is excited by signals with equal magnitude. The simulated S-parameters of the proposed array antenna structure is illustrated in Fig.4. And fig.4 and fig.5 shows the Reflection coefficient and radiation pattern of the proposed antenna Yagi-Uda antenna respectively.

As shown in Fig.3, all the 16 parasitic elements perform very consistently with better S11 and acceptable bandwidth to cover 3.5 $\mathrm{GHz}$.

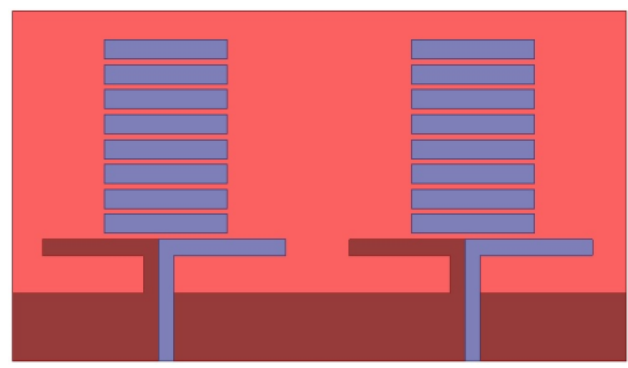

Fig. 3: Two Elements Linear Array Yagi-Uda Antenna

\section{Results and Discussions}

\subsection{Reflection coefficient and Current Distribution of Single Antenna}

The reflection coefficient for the eight elements single Yagi antenna is as shown below. The figure 4 depicts the S11 value of 36.2 with a band width of $500 \mathrm{Mhz}$.

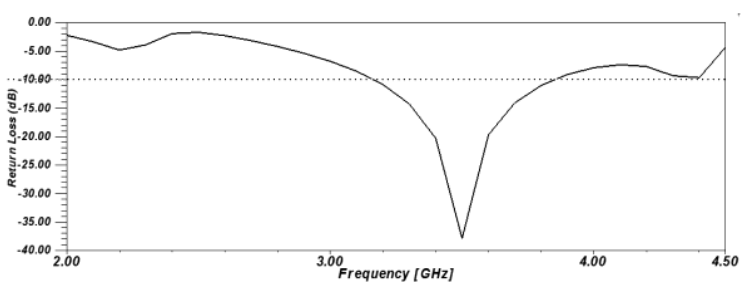

Fig. 4: Reflection Coefficient of Single Yagi-Uda Antenna 


\subsection{Radiation pattern of single 8 element Yagi antenna}

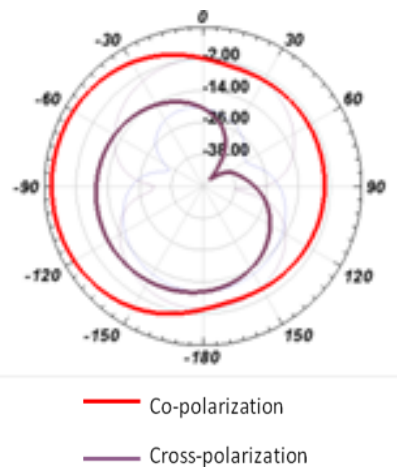

Fig. 5: Radiation Pattern of Yagi-Uda Antenna

It is observed from the figure 5 that the co polarization is more compared to cross polarization which is desirable quality of working of good antenna.

\subsection{Current Distribution of two element linear array}

In The Yagi-Uda array the surface travelling wave is described by the current distribution in each element and by the phase velocity of the travelling wave. In calculation of current distribution in the Yagi-Uda antenna where the currents are induced in the parasitic elements via the mutual impedances with the exciter, and radiation occurs as from a set of discrete sources. Green[10] has analyzed arrays with up to ten elements using this method with good results.

Current distribution in two element linear array at $3.5 \mathrm{GHz}$ is 6.4 $\mathrm{A} / \mathrm{m}$.

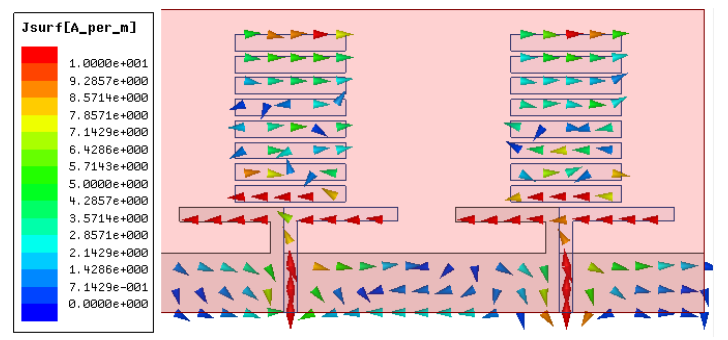

Fig. 6: Current Distribution in Two Element Linear Array

\subsection{Envelope Correlation Coefficient (ECC)}

Envelope Correlation Coefficient tells us how independent two antennas' radiation patterns are. So if one antenna was completely horizontally polarized, and the other was completely vertically polarized, the two antennas would have a correlation of zero. Similarly, if one antenna only radiated energy towards the sky, and the other only radiated energy towards the ground, these antennas would also have an ECC of 0. Hence, Envelope Correlation Coefficient takes into account the antennas' radiation pattern shape, polarization, and even the relative phase of the fields between the two antennas. The antenna structures in this study exhibit an envelope correlation coefficient of 0 at $3.5 \mathrm{GHz}$ as shown in Figure 7. And also it is below 0.5 for the entire bandwidth of the proposed antenna which shows better isolation between two antenna elements in array.

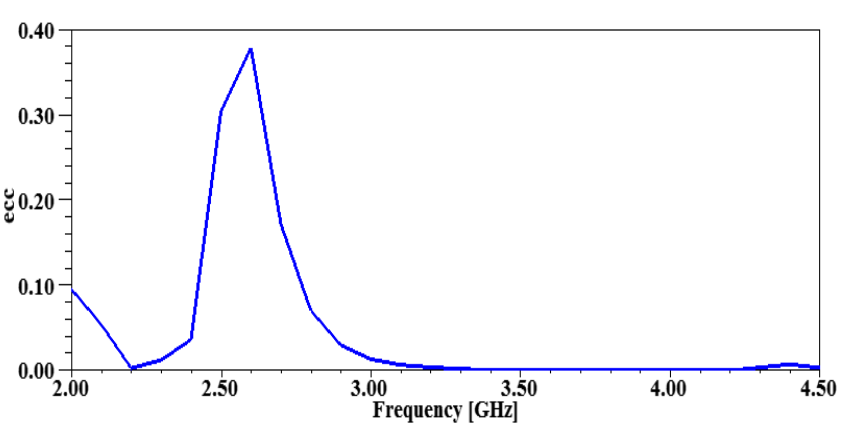

Fig. 7: Envelop Correlation Coefficient of Array Antenna

\section{Parametric Analysis}

All significant physical parameters, such as W5,W4,W3,L1,substrate materials should be adjusted carefully in order to achieve a good performance. In this section, the effects of these parameters on impedance bandwidth and realized gain are examined to facilitate the design of a same or homogeneous antenna with different specification.

\subsection{Different lengths of dipole director}

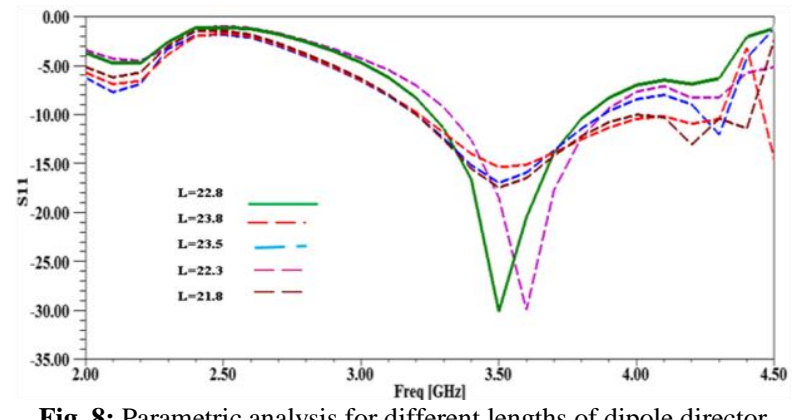

Fig. 8: Parametric analysis for different lengths of dipole director

The antenna which is modeled in the paper needs significance to optimize the length of the dipole driver, width of the driver, space between parasitic directors and substrate. The $\mathrm{S}$ parameters of the length of drivers were shown in figure8.Each value of the length is $23.8 \mathrm{~mm}, 24.3 \mathrm{~mm}, 22.8 \mathrm{~mm}, 22.3$, and $21.8 \mathrm{~mm}$. For length $23.8 \mathrm{~mm}$ and $\mathrm{S} 11$ at $3.5 \mathrm{GHz}$ is -15.3865 ,realized gain is 8.23 and bandwidth is $800 \mathrm{Mhz}$.

For length $24.3 \mathrm{~mm}$ and $\mathrm{S} 11$ at $3.5 \mathrm{GHz}$ is -16.9845 ,realizedgain is 8.65 and bandwidth is $600 \mathrm{Mhz}$. For length $22.3 \mathrm{~mm}$ and $\mathrm{S} 11$ at $3.5 \mathrm{GHz}$ is -17.48 ,realized gain is 8.59 and bandwidth is 800Mhz.For length $22.8 \mathrm{~mm}$ and $\mathrm{S} 11$ at $3.5 \mathrm{GHz}$ is -26.67 ,realized gain is 10.5 and bandwidth is $620 \mathrm{Mhz}$.For length $21.8 \mathrm{~mm}$ and $\mathrm{S} 11$ at $3.5 \mathrm{GHz}$ is -30.10 ,realized gain is 8.37 and bandwidth is $500 \mathrm{Mhz}$.

\subsection{Different widths of director dipole}

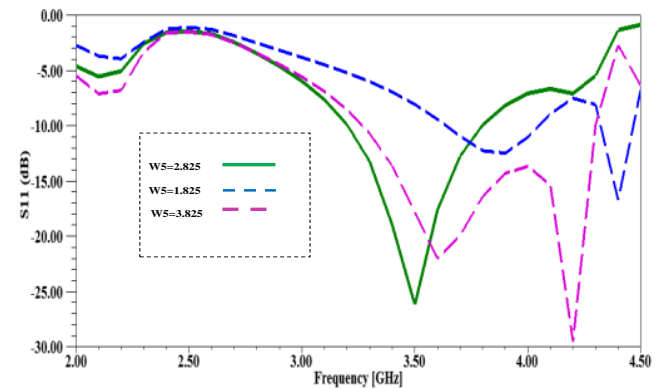

Fig. 9: Parametric analysis for different widths of director dipole

The $\mathrm{S}$ parameters of the width of directors were shown in figure 9 . Each value of the length is $2.825,3.825 \mathrm{~mm}$ and $1.825 \mathrm{~mm}$. From 
the simulation curve in figure 5, when the length is $3.825 \mathrm{~mm}$, the error of the curve is bigger.

\subsection{Parametric analysis of different substrate}

The changes in value of S11 parameters using different substrate materials were shown in figure 10 .

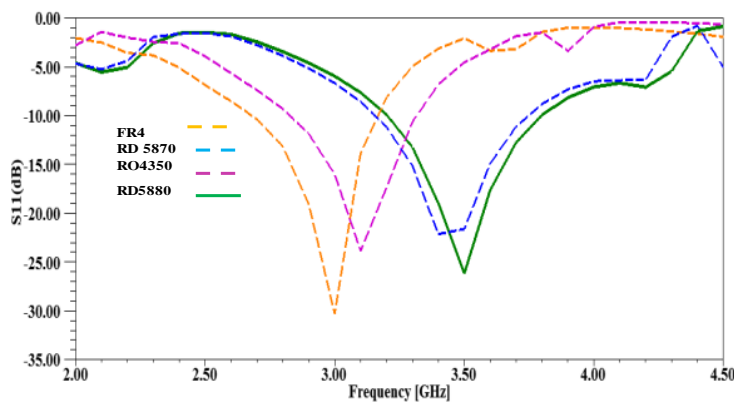

Fig. 10: Paramentric analysis for different substrate material

Each substrate exhibits its own properties by which S parameters, bandwidth and gain of the antenna changes accordingly. S11 of Rogers Duroid 5880 is found to be -26.18 with bandwidth of 600Mhz,S11 of Rogers Druroid 5870 is -21.046 with bandwidth of $500 \mathrm{Mhz}, \mathrm{S} 11$ of Rogers 4350 is -24.472 bandwidth 400Mhz ,S11 of Fr4 -32.24 with bandwidth of 390Mhz. From above analysis it is obvious that RT Duroid 5880 is providing greater bandwidth comparatively.

\subsection{Different spaces between parasitic patches}

The S parameters are optimized with different values but equal gap between parasitic patches of drivers were shown in figure 11 .

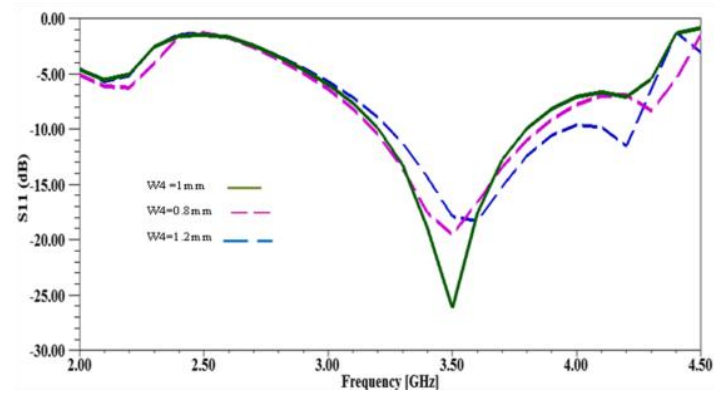

Fig. 11: Parametric analysis for different spaces between parasitic patches

Each value of the length $0.8 \mathrm{~mm}, 1 \mathrm{~mm}$ and $1.2 \mathrm{~mm}$. The S parameters of the space between the parasitic element are shown in figure 11. From the simulation curve in figure, when the length is $1.2 \mathrm{~mm}$ the error of the curve is bigger. The curve near center frequency with the distance $\mathrm{s}=1 \mathrm{~mm}$.

When width w4 is $1.2 \mathrm{~mm}$ then gain of antenna is 7.7 and bandwidth is $600 \mathrm{Mhz}$. When width w4 is $0.8 \mathrm{~mm}$ then gain of the antenna is 8.61 and bandwidth is $700 \mathrm{Mhz}$.

Table 2: Comparison with Previous Work

\begin{tabular}{|l|l|l|l|l|l|}
\hline Work & $\begin{array}{l}\text { Antenna } \\
\text { Size }\end{array}$ & $\begin{array}{l}\text { actua- } \\
\text { tors }\end{array}$ & Bandwidth & Gain & Frequency \\
\hline$[1]$ & $136 \times 68$ & $\begin{array}{l}\text { MIM } \\
\text { O }\end{array}$ & $200 \mathrm{Mhz}$ & NR & $3.5 \mathrm{GHz}$ \\
\hline$[11]$ & $100 \times 92.8$ & Yagi & $160 \mathrm{Mhz}$ & $\begin{array}{l}7.37 \mathrm{~d} \\
\mathrm{Bi}\end{array}$ & $5.38 \mathrm{GHz}$ \\
\hline$[13]$ & $263 \times 263$ & $\begin{array}{l}\text { MIM } \\
\text { O }\end{array}$ & $400 \mathrm{Mhz}$ & $\begin{array}{l}7 \\
\mathrm{~dB}\end{array}$ & $5.8 \mathrm{GHz}$ \\
\hline$[5]$ & $100 \times 80$ & Yagi & $560 \mathrm{Mhz}$ & $\begin{array}{l}9.5 \mathrm{~d} \\
\mathrm{Bi}\end{array}$ & $5.2 \mathrm{GHz}$ \\
\hline $\begin{array}{l}\text { Pro- } \\
\text { posed }\end{array}$ & $110 \times 60$ & Yagi & $\sim 600 \mathrm{Mhz}$ & $\begin{array}{l}10.6 \\
\mathrm{~dB}\end{array}$ & $3.5 \mathrm{GHz}$ \\
\hline
\end{tabular}

\section{Measures Result}

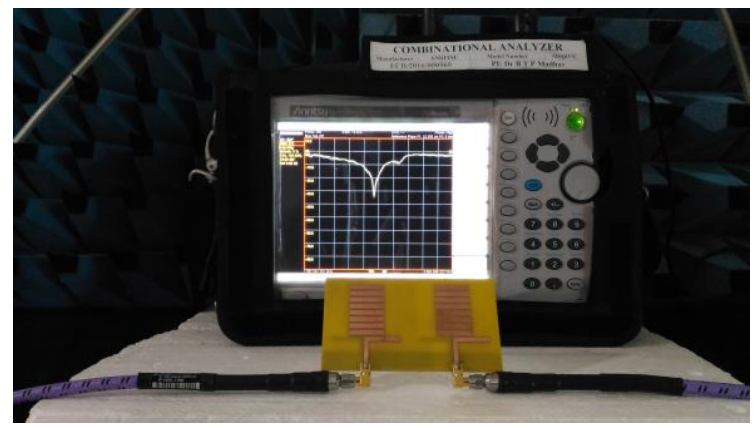

Fig 12. Measured data on VNA

\section{Conclusion}

In this paper, we proposed a two element linear array antenna, which is composed of sixteen microstrip Quasi-Yagi parasitic elements and forms a $2 \times 2$ linear structure with center frequency operating in the $5 \mathrm{G}$ low-band at $3.5 \mathrm{GHz}$. The simulation results show that the antenna array has high gain (peak value about $10.5 \mathrm{~dB}$ ), wide bandwidth about $600 \mathrm{MHz}$ at the center frequency of $3.5 \mathrm{GHz}$ and can approximately achieved. Meanwhile, within the operating bandwidth, good F/B ratios 13.68 has been observed. With this we can conclude that this proposed antenna has acquired almost the desirable characteristics which suits better for the vehicular antenna and fits better in $3.5 \mathrm{GHz}$ low band of $5 \mathrm{G}$.

\section{Acknowledgement}

The authors deeply express their gratitude to ALRC research centre, Department of ECE, KLEF for their encouragement during this work. Further, we express our gratitude to DST through ECR/2016/000569, FIST SR/FST/ETI-316/2012 and $\mathrm{EEQ/2016/000604.}$

\section{References}

[1] Eight-Element Antenna Array at $3.5 \mathrm{GHz}$ for MIMO Wireless Application, Progress In Electromagnetics Research C, Vol. 78, 209-216, 2017

[2] 2. A 4 Element Dual wideband circular Yagi MIMO Antenna System with Loop Excitation.

[3] Microstrip Magnetic Dipole Yagi Antenna with Enhanced Impedance

Bandwidth and Reduced Size for Wideband Wireless Applications Progress In Electromagnetics Research C, Vol. 73 , 105-113, 2017

[4] Huang, J. and A. C. Densmore, "Microstrip Yagi array antenna for mobile satellite vehicle application," IEEE Trans. Antennas and Propagat., Vol. 39, No. 7, 1024-1030, 1991.

[5] Liu, J., Y. Kang, J. Chen, \& Y. Long. 2014. Yagi array of microstrip quarter-wave patch antennas with microstrip lines coupling. Int. J. Antennas Propag. 2014:102362.

[6] Elliot, R. S., Antenna Theory and Design, Wiley, 2003.

[7] Lee, J. L. and J. Yeo, "Modified broadband Quasi-Yagi antenna with enhanced gain and bandwidth," Microwave Optical Technology Letters, Vol. 55, 406-409, 2013.

[8] Liu, J., Q. Xue, and Y. L. Long, "4-element Yagi array of microstrip quarter-wave patch antennas," Proc. IEEE International Wireless Symposium, 1-4, 2014.

[9] Hao, Y., G. Wang, Y. Tian, Y. Wang, L. Yu, and X. Ye, "Wide beamwidth circularly polarized microstrip Yagi array antenna," IEEE Conference on Communication Problem-Solving, 172174, 2015.

[10] G Jyothsna Devi, Reconfigurable Mimo Antenna For 5G Communication Applications, International Journal of Pure and Applied Mathematics, Vol 117, No. 18, 2017, pp 89-95. 
[11] B T P Madhav, T Venkateswara rao, T Anil Kumar, Design of 4Element Printed Array Antenna for Ultra-Wideband Applications, International Journal of Microwave and Optical Technology, Vol.13, No.1, January 2018, pp 8-17.

[12] D. Sreenivasa Rao, G. Lalitha, S. Mohammad Parvez, J. Naveen,

D. Mani Deepak, A. N. Meena Kumari, A Frequency Reconfigurable Spiral F-Shaped Antenna for Multiple Mobile Applications, Lecture Notes in Electrical Engineering, ISSN: 1876-1100, Vol 471, 2018, pp 571-580.

[13] D S Ramkiran, K Manikanta Varma, Y Sireesha, A CPW Fed Serrated Fractal Antenna for UWB Applications, International Journal of Pure and Applied Mathematics, Volume 117, No. 16 2017, pp 679-683.

[14] Y Usha Devi, M S S Rukmini, Triple Band CPW-Fed Monopole Antenna for GPS, LTE And Aeronautical Mobile Based IOT Applications, International Journal of Pure and Applied Mathematics, Volume 117, No. 18, 2017, pp 15-21.

[15] Vamseekrishna Allam, Defected Ground Structure Switchable Notch Band Antenna for UWB Applications, Smart Innovation, Systems and Technologies, Vol 77, pp 139-145, 2018.

[16] S S Mohan Reddy, P Mallikarjuna rao, Asymmetric Defected Ground Structured Monopole Antenna for Wideband Communication Systems, International Journal of Communications Antenna and Propagation, ISSN: 2039-5086, Vol 5, Issue 5, Dec-15, pp 256-262.

[17] D S Ram Kiran, Novel compact asymmetrical fractal aperture Notch band antenna, Leonardo Electronic Journal of Practices and Technologies, ISSN 1583-1078, Vol 27, Issue 2, December 2015, pp 1-12.

[18] M S S S Srinivas, T V Ramakrishna, Bandwidth enhanced electromagnetic bandgap structure structured closed ground monopole antenna, Leonardo Electronic Journal of Practices and Technologies, ISSN 1583-1078, Issue 28, June 2016, pp 211 224.

[19] B. T. P. Madhav, A. V. Chaitanya, R. Jayaprada, M. Pavani, Circular Monopole Slotted Antenna with FSS for High Gain Applications, ARPN Journal of Engineering and Applied Sciences, ISSN 1819-6608, Vol. 11, No. 15, August 2016, pp 17.

[20] G. V. Krishna, Bandwidth Enhanced Antipodal Vivaldi Antenna for Wide Band Communication Applications, Indian Journal of Science and Technology, ISSN: 0974-6846, Vol 9, Issue 31, August 2016, pp 1-6. 